\section{Estimación del impacto del consumo de sal en los niveles de presión arterial en población no hipertensa entre 15 y 64 años}

\author{
KAREN DOMÍNGUEZ CANCINO ${ }^{1, \mathrm{a}, \mathrm{b}}$, \\ MARÍA CRISTINA PAREDES ESCOBAR ${ }^{2, a, c}$
}

\section{Impact of high salt consumption of blood pressure on a non-hypertensive population}

Background: There is conflicting evidence regarding the role of salt intake in blood pressure (BP). Aim: To estimate the impact of salt consumption on the BP level of a non-hypertensive population aged between 15 and 64 years. Material and Methods: Analytical-observational study using data from the National Health Survey 2009-2010. A BP cut-off point at 120/80 mmHg BP was considered to determine risk. Salt consumption was divided into four strata. The prevalence ratios (PR) were determined using the Poisson model with robust variance. The formulas of the studies of Dal Grande and Walter for the estimation of population attributable fraction (PAF) were used. Results: The sample was constituted by 1,263 individuals and $24.3 \%$ had BP at risk. A statistically significant association was observed between high salt intake and risk $B P$ with PR of 1.91 (95\% confidence intervals (CI) 1.44-2.57) in the consumption stratum of $11 \mathrm{~g} /$ day and more. It was estimated that $4.7 \%$ (95\% CI 4.2-5.2) of $B P$ risk can be attributed to salt consumption, when controlling by age group, sex and educational level. Conclusions: The 4.7\% PAF is lower than the figure of $30 \%$ reported abroad. Interventions to reduce salt consumption in the entire population and the identification of risk groups are recommended.

(Rev Med Chile 2017; 145: 1597-1604)

Key words: Arterial Pressure; Public Health; Sodium, Dietary.
'Escuela de Enfermería,

Universidad FinisTerrae. Santiago,

Chile.

Escuela de Enfermería,

Universidad Bernardo O'Higgins.

Santiago, Chile.

${ }^{a}$ Enfermera Estudiante de doctorado en Salud Pública, Universidad de Chile.

${ }^{b}$ Magíster en Salud Pública

'Magíster en Bioética.

Las autoras declaran no tener conflictos de interés.

Trabajo no recibió financiamiento.

Recibido el 22 de mayo de 2017, aceptado el 26 de diciembre de 2017.

Correspondencia a: Karen Domínguez Cancino Escuela de Enfermería, Universidad Finis Terrae. Avenida Pedro de Valdivia \#1509. Santiago, Chile. kdominguez@uft.cl egún datos de la Organización Mundial de la Salud (OMS), la hipertensión arterial (HTA) causó 9,4 millones de muertes a nivel mundial en el año $2010^{1}$. Reducir los niveles de presión arterial (PA) por debajo de 140/90 $\mathrm{mmHg}$ se asocia con una reducción de las complicaciones cardiovasculares $^{2}$. En algunos grupos etarios, el riesgo de sufrir enfermedades cardiovasculares se duplica por cada incremento de $20 / 10 \mathrm{mmHg}$ de la PA, considerando el inicio del riesgo en valores de PA de 115/75 $\mathrm{mmHg}^{2}$, evidenciando la importancia de generar intervenciones para el control de la PA en población no hipertensa .
Existe variada evidencia que indica que la reducción del consumo de sal $(\mathrm{NaCl})$ disminuye los niveles de $\mathrm{PA}^{3,4}$. Estudios clínicos han demostrado que la reducción del consumo de sal en $1 \mathrm{~g} /$ día genera una reducción de la PA de 1/0,5 $\mathrm{mmHg}$ en hipertensos, y de $0,5 / 0,25 \mathrm{mmHg}$ en normotensos ${ }^{3-5}$.

Organismos internacionales indican que 30\% de la PA en rangos alterados $(\geq 140 / 90 \mathrm{mmHg}$ ) estaría atribuida al consumo de sal, estimación que proviene de un único estudio ${ }^{6}$.

De manera contraria, algunos investigadores indican que no existiría dicha relación e incluso 
mencionan que una reducción marcada del consumo de sal en un periodo corto de tiempo sería perjudicial $^{3,5,7-9}$. En la revisión Cochrane de $\mathrm{He}, \mathrm{Li}$ y MacGregor sobre los efectos a largo plazo de la reducción modesta del consumo de sal en la PA del año 2013, se destacan dos artículos publicados en el Journal of the American Medical Association, donde afirman que un menor consumo de sal se asocia a una mayor mortalidad cardiovascular, existiendo una asociación entre ingesta de sal y riesgo cardiovascular en forma de $\mathrm{J}^{5}$. A pesar que estos estudios han sido criticados por fallas metodológicas, este tipo de información abre el tema de la variabilidad en las respuesta de la PA de los individuos frente a un consumo elevado de sal ${ }^{3-5,8,10,11}$.

Dado lo anterior, resulta fundamental esclarecer la asociación entre consumo de sal y PA, en especial en personas no hipertensas. El objetivo de este estudio es estimar el impacto del consumo de sal en el nivel de presión arterial de población no hipertensa entre 15 y 64 años.

\section{Material y Métodos}

\section{Diseño de estudio}

Estudio de corte transversal, analítico-observacional, realizado en base a los datos de la Encuesta Nacional de Salud de 2009-2010 (ENS 2009-2010).

\section{Población y muestra}

La población estuvo compuesta por 5.293 individuos mayores de 15 años, que correspondía a la muestra disponible en la base de datos de la ENS facilitada por el Departamento de Información y Estadística en Salud (DEIS) del Ministerio de Salud de Chile.

Criterios de inclusión y exclusión: Se incluyeron a todos los individuos que contaban datos registrados en la base de datos facilitada por el DEIS. Se excluyeron a los mayores de 64 años, personas con diagnóstico o sospecha de HTA (la sospecha de HTA se define como presentar valores de $\mathrm{PA} \geq 140 / 90$ en base a las mediciones realizadas por profesionales dispuestos para la ENS, el procedimiento se detallada más adelante), haber sido informado de presentar HTA por un profesional de salud, uso de fármacos para controlar la patología o tener cambios en el estilo de vida (como reducción del consumo de sal). Además, se eliminaron aquellos individuos que contaban con datos incompletos

\section{Variables}

Las principales variables del estudio fueron el consumo de sal y la PA. El consumo de sal fue medido a través de una muestra aislada de orina utilizando la fórmula de Tanakata para la estimación de excreción de sodio de $24 \mathrm{~h}^{12}$. Con respecto a la PA, se utilizó un protocolo estandarizado de 3 tomas de PA promediadas, realizado por profesionales capacitados, haciendo uso de un aparato de presión automático (Omron HealthCare, modelo HEM $742^{\circledR}$, Japón $)^{13}$. La PA fue estimada como de riesgo o sin riesgo tomando como punto de corte la $\mathrm{PA} \geq 120 / 80 \mathrm{mmHg}$, de esta manera se conforman 2 grupos, el grupo de personas con valores de $\mathrm{PA}<120 / 80 \mathrm{mmHg}$ reconocido como "sin riesgo" $y$ un grupo con PA con valores $\geq 120 / 80 \mathrm{~mm} \mathrm{Hg}$ y $<140 / 90 \mathrm{mmHg}$, identificado como "con riesgo". Además, se analizaron variables sociodemográficas: edad, sexo, nivel económico y zona geográfica de residencia, y clínicas de los individuos: estado nutricional, consumo de tabaco, consumo de alcohol, colesterol total, triglicéridos, consumo de pescado, consumo de cereales, consumo de lácteos, consumo de frutas y verduras, presencia de diabetes mellitus, enfermedad renal crónica y actividad física; la forma en que estas fueron trabajadas se presenta en las Tablas 2 y 3.

\section{Plan de análisis}

Se realizó estadística descriptiva a través promedios y cuartiles (p25-p75) para variables cuantitativas, y porcentajes para variables cualitativas. Se realizó estadística inferencial bivariada, para establecer asociaciones y correlaciones entre las variables principales y otras variables sociodemográficas y clínicas $(\alpha=0,05)$. Se determinaron las razones de prevalencia (RP) a través del modelo Poisson con varianza robusta, crudos y ajustados por variables de confusión. Los confusores fueron identificados en base a criterio teórico y estadístico $(\mathrm{p}>0,1)$. Para la estimación de la fracción atribuible poblacional (FAP) y los intervalos de confianza se utilizó las fórmulas de los estudios de Dal Grande y Walter (Tabla 1), respectivamente ${ }^{14,15}$. Los análisis se realizaron a través del software estadístico STATA versión 12.0, disponible en la Facultad de Medicina de la Universidad de Chile. 
Estimación de impacto del consumo de sal en presión arterial - K. Domínguez Cancino et al

Tabla 1. Fórmulas utilizadas en la estimación de fracción atribuible desde estudios de corte transversal

\begin{tabular}{|lll|}
\hline \multicolumn{2}{|c|}{ Estimaciones } & Fórmulas \\
\hline 1 & Fracción atribuible poblacional & $F A P=\mathrm{pd} \frac{(R P-1)}{R P}$ \\
2 & Error estándar & $\mathrm{s}=\frac{S E(\mathrm{PÂR})}{(1-\mathrm{PÂR})}$ \\
\hline 3 & Fracción atribuible límite inferior & $P A R l=1-\exp (\operatorname{Ln}[1-\mathrm{PÂR}]+1,96 \mathrm{~s})$ \\
\hline 4 & Fracción atribuible límite superior & $P A R u=1-\exp (\operatorname{Ln}[1-\mathrm{PÂR}]-1,96 \mathrm{~s})$ \\
\hline
\end{tabular}

*FAP: fracción atribuible poblacional; pd: proporción de casos expuestos al riesgo; RR: riesgo relativo; SE: error estándar del modelo; S: error estándar; PARI: fracción atribuible poblacional límite inferior; PARu: fracción atribuible poblacional límite superior.

Tabla 2. Características sociodemográficas de la población de estudio de acuerdo a nivel de presión arterial $(n=1.263)$

\begin{tabular}{|c|c|c|c|}
\hline Características sociodemográficas & $\begin{array}{l}\text { Presión arterial normal } \\
\quad(<120 / 80 \mathrm{mmHg})\end{array}$ & $\begin{array}{l}\text { Presión arterial de riesgo } \\
\quad(\geq 120 / 80 \mathrm{mmHg})\end{array}$ & $p$ value \\
\hline $\begin{array}{l}\text { Edad en estratos, } n \text { (\%) } \\
\text { 15-24 años } \\
\text { 25-44 años } \\
\text { 45-64 años }\end{array}$ & $\begin{array}{l}180(18,8) \\
511(53,5) \\
265(27,7)\end{array}$ & $\begin{array}{r}22(7,2) \\
134(43,6) \\
151(49,2)\end{array}$ & $<0,0001$ \\
\hline $\begin{array}{l}\text { Sexo, N (\%) } \\
\text { Masculino } \\
\text { Femenino }\end{array}$ & $\begin{array}{l}348(36,4) \\
608(63,6)\end{array}$ & $\begin{array}{l}191(62,2) \\
116(37,8)\end{array}$ & $<0,0001$ \\
\hline $\begin{array}{l}\text { Nivel educativo, n (\%) } \\
\text { Bajo (< } 8 \text { años) } \\
\text { Medio (8-12 años) } \\
\text { Alto (> } 12 \text { años) }\end{array}$ & $\begin{array}{l}115(12,0) \\
574(60,0) \\
267(27,9)\end{array}$ & $\begin{array}{r}58(18,9) \\
188(61,2) \\
61(19,9)\end{array}$ & $<0,001$ \\
\hline $\begin{array}{l}\text { Nivel económico, } n(\%) \\
\text { Menos de } \$ 136.999 \\
137.000 \text { a } \$ 180.999 \\
181.000 \text { a } \$ 250.999 \\
251.000 \text { a } \$ 350.999 \\
\$ 351.000 \text { a } \$ 450.999 \\
\$ 451.000 \text { a } \$ 650.999 \\
\$ 651.000 \text { o más }\end{array}$ & $\begin{array}{l}138(14,4) \\
143(15,0) \\
169(17,7) \\
137(14,3) \\
122(12,8) \\
102(10,7) \\
145(15,1)\end{array}$ & $\begin{array}{r}47(15,3) \\
52(16,9) \\
58(18,9) \\
40(13,0) \\
29(9,5) \\
31(10,1) \\
50(16,3)\end{array}$ & 0,733 \\
\hline $\begin{array}{l}\text { Zona geográfica de residencia, n (\%) } \\
\text { Norte } \\
\text { Centro } \\
\text { Sur }\end{array}$ & $\begin{array}{l}300(31,4) \\
212(22,2) \\
444(46,4)\end{array}$ & $\begin{array}{r}84(27,4) \\
75(24,4) \\
148(48,2)\end{array}$ & 0,385 \\
\hline
\end{tabular}

\section{Aspectos éticos}

Con respecto a los aspectos éticos, estos provienen de las consideraciones tomadas en la ENS a cargo de la Pontificia Universidad Católica de Chile. Se obtuvo autorización para utilizar la base de datos secundaria por parte del DEIS.

\section{Resultados}

\section{Población y muestra}

La muestra estuvo compuesta por 1.263 individuos. Se mantuvieron los individuos que tuvieron variables de interés para el estudio $(n=3.637$ per- 
Tabla 3. Características clínicas de la población de estudio $(\mathbf{n}=\mathbf{1 . 2 6 3})$

\begin{tabular}{|c|c|c|c|}
\hline Características clínicas & $\begin{array}{c}\text { Presión arterial } \\
\text { normal } \\
(<120 / 80 \mathrm{mmHg})\end{array}$ & $\begin{array}{c}\text { Presión arterial } \\
\text { de riesgo } \\
(\geq 120 / 80 \mathrm{mmHg})\end{array}$ & $p$ value \\
\hline $\begin{array}{l}\text { Estado nutricional (IMC), n (\%) } \\
\text { Normal } \\
\text { Enflaquecido } \\
\text { Sobrepeso } \\
\text { Obeso } \\
\text { Obeso mórbido }\end{array}$ & $\begin{array}{r}362(37,9) \\
13(1,4) \\
370(38,7) \\
201(21,0) \\
10(1,0)\end{array}$ & $\begin{array}{r}67(21,8) \\
2(0,7) \\
135(44,0) \\
95(30,9) \\
8(2,6)\end{array}$ & $<0,0001$ \\
\hline $\begin{array}{l}\text { Consumo de tabaco, } \mathrm{n}(\%) \\
\text { Fumador actual } \\
\text { Ex fumador }(<6 \text { meses) } \\
\text { Ex fumador }(>6 \text { meses) } \\
\text { Nunca fumador }\end{array}$ & $\begin{array}{r}438(45,8) \\
34(3,6) \\
153(16,0) \\
331(34,6)\end{array}$ & $\begin{array}{r}130(42,4) \\
9(2,9) \\
50(16,3) \\
118(38,4)\end{array}$ & 0,604 \\
\hline $\begin{array}{l}\text { Consumo de alcohol (puntaje AUDIT), n (\%) } \\
\text { 0-7 puntos } \\
8-15 \text { puntos } \\
16-19 \text { puntos } \\
20 \text { o más puntos }\end{array}$ & $\begin{array}{rr}844 & (88,3) \\
90 & (9,4) \\
12 & (1,3) \\
10 & (1,0)\end{array}$ & 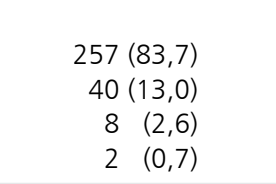 & 0,087 \\
\hline $\begin{array}{l}\text { Colesterol total, n (\%) } \\
\quad<200 \mathrm{mg} / \mathrm{dl} \\
\geq 200 \mathrm{mg} / \mathrm{dl}\end{array}$ & $\begin{array}{l}572(65,8) \\
298(34,2)\end{array}$ & $\begin{array}{l}111(40,4) \\
164(59,6)\end{array}$ & $<0,0001$ \\
\hline $\begin{array}{l}\text { Colesterol HDL, N (\%) } \\
\geq 40 \mathrm{mg} / \mathrm{dl} \\
<40 \mathrm{mg} / \mathrm{dl}\end{array}$ & $\begin{array}{l}649(74,8) \\
219(25,2)\end{array}$ & $\begin{array}{r}193(70,2) \\
82(29,8)\end{array}$ & 0,132 \\
\hline $\begin{array}{l}\text { Triglicéridos, n (\%) } \\
\quad<150 \mathrm{mg} / \mathrm{dl} \\
\geq 150 \mathrm{mg} / \mathrm{dl}\end{array}$ & $\begin{array}{l}631(72,5) \\
239(27,5)\end{array}$ & $\begin{array}{l}149(54,2) \\
126(45,8)\end{array}$ & $<0,0001$ \\
\hline $\begin{array}{l}\text { Consumo de pescado, } \mathrm{n}(\%) \\
\text { Menos de una vez por semana } \\
\text { Al menos una vez por semana }\end{array}$ & $\begin{array}{l}420(43,9) \\
536(56,1)\end{array}$ & $\begin{array}{l}129(42,0) \\
178(58,0)\end{array}$ & 0,556 \\
\hline $\begin{array}{l}\text { Consumo de cereales, n (\%) } \\
\text { Al menos } 1 \text { porción por día } \\
\text { Menos de } 1 \text { porción por día }\end{array}$ & $\begin{array}{l}130(13,6) \\
826(86,4)\end{array}$ & $\begin{array}{rr}20 & (6,5) \\
287 & (93,5)\end{array}$ & 0,001 \\
\hline $\begin{array}{l}\text { Consumo de lácteos, n (\%) } \\
\text { Menos de } 1 \text { vez por día } \\
\text { Al menos } 1 \text { vez por día }\end{array}$ & $\begin{array}{l}509(53,2) \\
447(46,8)\end{array}$ & $\begin{array}{l}137(44,6) \\
170(55,4)\end{array}$ & 0,009 \\
\hline $\begin{array}{l}\text { Consumo de frutas y verduras, n (\%) } \\
5 \text { porciones o más } \\
\text { Menos de } 5 \text { porciones }\end{array}$ & $\begin{array}{r}84(10,2) \\
737(89,8)\end{array}$ & $\begin{array}{r}34(12,9) \\
229(87,1)\end{array}$ & 0,222 \\
\hline $\begin{array}{l}\text { Diabetes mellitus, n (\%) } \\
\text { Menos de } 126 \mathrm{mg} / \mathrm{dl} \\
\text { Más de } 126 \mathrm{mg} / \mathrm{dl}\end{array}$ & $\begin{array}{r}941(98,4) \\
15 \quad(1,6)\end{array}$ & $\begin{array}{r}292(95,1) \\
15 \quad(4,9)\end{array}$ & 0,001 \\
\hline $\begin{array}{l}\text { ERC (Etapa 3), n (\%) } \\
\text { Si } \\
\text { No }\end{array}$ & $\begin{array}{rr}5 & (0,5) \\
951 & (99,5)\end{array}$ & $\begin{array}{r}1(0,3) \\
306(99,7)\end{array}$ & 0,662 \\
\hline $\begin{array}{l}\text { Actividad física (según instrumento GPAQ), n (\%) } \\
\text { Bajo } \\
\text { Moderado } \\
\text { Alto }\end{array}$ & $\begin{array}{l}244(25,5) \\
198(20,7) \\
514(53,7)\end{array}$ & $\begin{array}{c}80(26,1) \\
52(16,9) \\
175(57,00)\end{array}$ & 0,342 \\
\hline
\end{tabular}


sonas). Se excluyeron 929 personas mayores de 64 años, 1.335 con diagnóstico de HTA, con consumo de fármacos para su control o con cambios en el estilo de vida. Se eliminaron 110 individuos por tener datos incompletos.

La muestra estuvo constituida principalmente por personas de sexo femenino $(57,2 \%)$, con edad entre 25 y 44 años $(50,4 \%)$, con 12 años o menos de educación formal $(74,4 \%)$, ingreso menor a $\$ 351.000(63,1 \%)$, residentes de la zona sur del país $(47,3 \%)$.

\section{Variables principales de estudio}

Con respecto a la $\mathrm{PA}, 24,31 \%$ presentó valores $\geq 120 / 80 \mathrm{mmHg}$. El grupo estuvo compuesto por personas de mayor edad (49,2\% presentaban edades entre 45 y 64 años versus $27,7 \%$ del grupo por PA sin riesgo), sexo masculino (62,2\% versus $36,4 \%)$, y con un nivel educativo menor $(18,9 \%$ presentaba menos de 8 años de estudio versus $12,0 \%)$, presentando diferencias estadísticamente significativas con el grupo de comparación (Tabla 2).

El grupo de riesgo presentó un consumo mayor de sal (promedio: 10,29 g/día; p25-p75: 8,5311,95 ) versus el grupo con PA normal (promedio: 9,31 g/día; p25-p75: 7,87-10,61), siendo esta diferencia estadísticamente significativa (Figura 1).

\section{Características clínicas de la población de estudio}

Con respecto a las características clínicas, se encontraron diferencias estadísticamente significativas en estado nutricional, niveles de colesterol, triglicéridos, prevalencia de diabetes mellitus, con- sumo de cereales integrales y lácteos. El grupo de riesgo presentó mayor porcentaje de diagnóstico nutricional de exceso, colesterol y triglicéridos elevados, prevalencia de diabetes mellitus, menor porcentaje de consumo de cereales integrales y mayor consumo de lácteos (Tabla 3 ).

\section{Razón de prevalencia}

En relación a la razón de prevalencia de PA $\geq 120 / 80 \mathrm{mmHg}$ de acuerdo al nivel de consumo de sal, se observó que el grupo expuesto a un consumo de sal mayor a $11 \mathrm{~g} /$ día presentó un aumento de $91 \%$ de la prevalencia de PA de riesgo en comparación con los individuos que presentaban un consumo de sal menor a 8 g/día, esto controlado por edad, sexo y nivel educativo. Los valores de RP crudo y ajustado eran cercanos en los estratos inferiores de consumo, distanciándose en los más altos (Tabla 4). Con respecto al menor riesgo de presentar una PA considerada de riesgo en el grupo de personas que consumía menos de $8 \mathrm{gr} /$ día de sal, se observó que existía una reducción del $50 \%$ de la prevalencia del PA de riesgo al comparar con el estrato de consumo de $11 \mathrm{gr} /$ día o más (datos no presentados). Respecto a las variables de ajuste, se observó que el aumento de los años de vida, el sexo masculino y un bajo nivel educativo se comportaron como factores de riesgo en el aumento de los niveles de PA.

\section{Fracción atribuible poblacional}

En la Tabla 5 se presenta la fracción atribuible poblacional (FAP) del consumo de sal en la PA de riesgo. Se observó que 4,7\% (IC95\% 4,2-5,2) de la PA de riesgo estaría explicada por el consumo

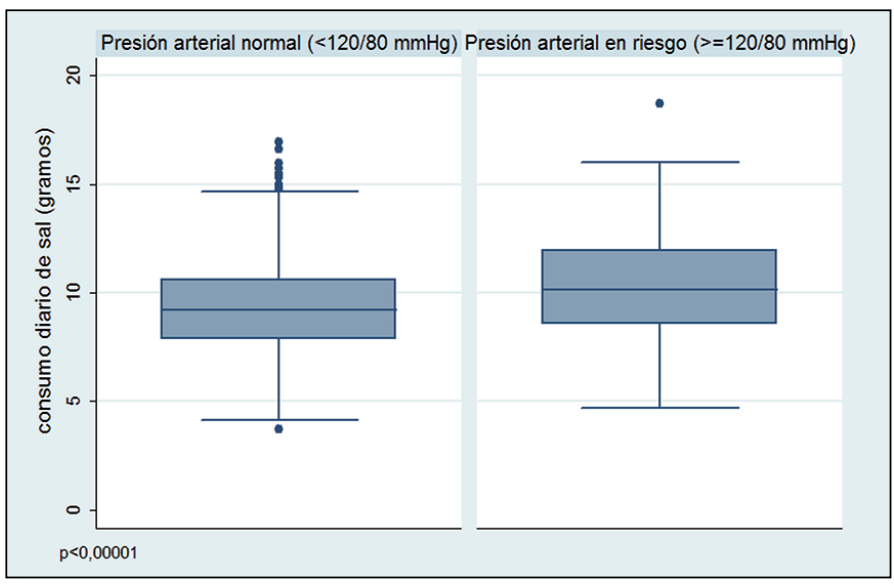

Figura 1. Consumo de sal (g/día) según categoría de presión arterial. 
Tabla 4. Razones de prevalencia (RP) crudos y ajustados e intervalos de confianza (95\%) de presión arterial de riesgo ( $\geq 120 / 80 \mathrm{mmHg}$ )

\begin{tabular}{|c|c|c|}
\hline & RP crudo (IC95\%) & RP ajustado* \\
\hline $\begin{array}{l}\text { Consumo de sal } \\
\text { Estrato } 1 \text { (0-8 g/día) } \\
\text { Estrato } 2 \text { (8-9,5 g/día) } \\
\text { Estrato } 3 \text { (9,5-11 g/día) } \\
\text { Estrato } 4 \text { (11 g/día y más) } \\
\text { Global }\end{array}$ & $\begin{array}{l}1 \quad \text { (referencia) } \\
1,21(0,86-1,68) \\
1,55(1,12-2,14) \\
2,29(1,70-3,010) \\
1,33(1,22-1,46)\end{array}$ & $\begin{array}{lr}1 & \text { (referencia) } \\
1,23 & (0,89-1,69) \\
1,34 & (0,98-1,83) \\
1,91 & (1,44-2,57) \\
1,24 & (1,13-1,35)\end{array}$ \\
\hline $\begin{array}{l}\text { Edad } \\
\qquad \begin{array}{l}\text { 15-24 años } \\
\text { 25-44 años } \\
45-64 \text { años }\end{array}\end{array}$ & $\begin{array}{ll}1 & \text { (referencia) } \\
1,91 & (1,25-2,91) \\
3,33 & (2,20-5,04)\end{array}$ & $\begin{array}{ll}1 & \text { (referencia) } \\
1,79 & (1,18-2,72) \\
2,91 & (1,92-4,39)\end{array}$ \\
\hline $\begin{array}{l}\text { Sexo } \\
\text { Masculino } \\
\text { Femenino }\end{array}$ & $\begin{array}{lr}1 & (\text { referencia }) \\
0,45 & (0,37-0,55)\end{array}$ & $\begin{array}{ll}1 & (\text { referencia }) \\
0,48 & (0,39-0,58)\end{array}$ \\
\hline $\begin{array}{l}\text { Nivel educativo } \\
\text { Bajo (< } 8 \text { años) } \\
\text { Medio ( } 8-12 \text { años) } \\
\text { Alto ( }>12 \text { años) }\end{array}$ & $\begin{array}{l}1 \quad \text { (referencia) } \\
0,74(0,58-0,94) \\
0,55(0,41-0,76)\end{array}$ & $\begin{array}{ll}1 & \text { (referencia) } \\
0,97 & (0,77-1,23) \\
0,84 & (0,62-1,14)\end{array}$ \\
\hline
\end{tabular}

*Ajustado por edad, sexo y nivel educativo.

Tabla 5. Fracción atribuible de presión arterial de riesgo al consumo de sal

\begin{tabular}{|c|c|c|}
\hline Consumo de sal & FAP\% crudo (IC95\%) & FAP\% Ajustado (IC95\%)* \\
\hline Estrato 1 (0-8 g/día) & 1 & 1 \\
\hline Estrato 2 (8-9,5 g/día) & $1,0 \% \quad(0,6-1,3)$ & $1,1 \% \quad(0,6-1,4)$ \\
\hline Estrato 3 (9,5-11 g/día) & $2,1 \% \quad(1,0-3,1)$ & $1,5 \% \quad(0,9-2,1)$ \\
\hline Estrato 4 (11 g/día y más) & $5,0 \% \quad(1,6-8,5)$ & $4,3 \% \quad(1,9-6,7)$ \\
\hline Global & $6,0 \% \quad(5,3-6,8)$ & $4,7 \% \quad(4,2-5,2)$ \\
\hline
\end{tabular}

*Ajustado por edad, sexo y nivel educativo.

de sal, existiendo un aumento de la FAP en la medida que aumenta el estrato de consumo de sal, triplicándose en el paso del estrato 3 al 4.

\section{Discusión}

Esta investigación tuvo por objetivo estimar el impacto del consumo de sal en los niveles de presión arterial $\geq 120 / 80 \mathrm{mmHg}$ en población no hipertensa entre 15 y 64 años. Se estima que 4,7\%(IC95\% 4,2-5,2) de la PA considerada de riesgo cardiovascular estaría explicada por el consumo de sal.

Dentro de los resultados obtenidos, se destaca el alto consumo de sal tanto en las personas con PA con o sin riesgo, que a pesar de presentar diferencias estadísticamente significativas, en términos clínicos resultan ser muy cercanos; se destaca también la relación dosis-respuesta observada, con valores significativos de RP y FAP en el estrato de mayor consumo de sal. Este hallazgo concuerda con investigaciones que muestran un alto consumo promedio generalizado en la población y una relación dosis-respuesta entre la reducción de la ingesta de sal y la caída de $\mathrm{PA}^{5,16,17}$.

La FAP obtenida en esta investigación dista de $30 \%$ referido a nivel internacional. Esto puede estar relacionado con diferentes aspectos: población utilizada para la estimación, tipo de estudio y variabilidad en la respuesta de la PA al consumo de sal. Con respecto al primer punto, variada 
literatura demuestra que la población hipertensa presenta un mayor beneficio en términos de PA que los normotensos al reducir su consumo de sal, población que no fue considerada en este estudio $^{3,5,16,17}$. En segundo lugar, los valores de reducción de PA del estudio de Joffres fueron extraídos de los metaanálisis de la Cochrane Review (He el al, 2004) y de Law et al (2003), indicando que una disminución del consumo de sodio de casi 2 g/día generaba una reducción de PA de 5/3 $\mathrm{mmHg}$. Al extrapolar estos datos a la encuesta nacional de Canadá, los investigadores concluyen que existe una reducción de $30 \%$ de la prevalencia de HTA en términos relativos (reducción de prevalencia de $19,4 \%$ a $13,5 \%)^{6}$. Se destaca que las estimaciones utilizan diferentes supuestos y se realizan con fuentes de información disímiles, sumado a que corresponde a una medida relativa y no absoluta. Finalmente, la respuesta diferencial entre individuos ha sido explicada por el fenómeno conocido como sensibilidad a la sal, concepto que presenta varias complejidades en su definición ${ }^{18-20}$, mecanismos que explican este fenómeno, forma de medir ${ }^{18,21}$ y categorías de respuesta ${ }^{19,20}$. Dos de las tres variables identificadas como confusores se asocian al concepto anterior. La edad, por una parte, ha sido identificada como uno de los factores que generan variabilidad en la respuesta de la PA ante el consumo de sal. Este efecto es explicado por cambios conformacionales de vasos sanguíneos y funcionamiento cardiaco propio del envejecimiento ${ }^{22}$. En el caso del sexo, las diferencias han sido explicadas por efectos genéticos y hormonales ${ }^{23,24}$.

Con respecto al nivel educacional, las diferencias encontradas se han explicado desde la perspectiva de determinantes sociales, reconociéndose la existencia de un gradiente social ${ }^{1}$.

Este estudio presenta limitaciones. En primer lugar la alta prevalencia de consumo excesivo de sal obligó a trabajar con estratos de consumo diferentes a los recomendados por la OMS ${ }^{1}$. Sumado a lo anterior, existen dos aspectos relacionados a la estructura de la muestra de la ENS 2009-2010 que podrían afectar a los resultados: el muestreo complejo y el cálculo muestral en base a varianza máxima. En el primer caso, a pesar de no respetar el muestreo considerado en la ENS, por los criterios de inclusión y exclusión, el muestreo complejo, estratificado polietápico por conglomerados genera un aumento de la homogeneidad dentro de los grupos y la heterogeneidad entre grupos, lo que podría dar mayor variabilidad a los resultados. Además, la eliminación de las edades extremas de la muestra generó un sesgo de selección, razón por la cual los hallazgos no pueden ser generalizados a la población chilena en su totalidad.

Por otra parte, la utilización de varianza máxima, podría estar subestimando la PA alterada por estratos, considerando que fue de alrededor de $25 \%$ y no $50 \%$ como indica el supuesto. Sumado a lo anterior, se debe considerar el aumento en el error dado el efecto multiplicativo de los diferentes errores aleatorios considerados en la estructura de la muestra original.

La estimación de la FAP también es un tema a considerar, ya que se realizó considerando un estudio de corte transversal, haciendo uso de RP y no de RR, desconociendo la duración de la condición de estudio y temporalidad de la asociación, por lo que los resultados deben ser analizados con cautela ${ }^{25}$.

Como fortalezas, este estudio es la primera iniciativa conocida disponible en la literatura chilena que intenta estimar la FAP en esta relación y en un estudio de corte transversal, contando con un buen tamaño de muestral y medición de biomarcadores considerados como buenos indicadores. Se destaca la utilización de modelos estadísticos adecuados para los fines establecidos. Desde la mirada de salud pública, este tipo de estimaciones permiten identificar el enfoque con el que se debe abordarlos factores de riesgo cardiovascular. De acuerdo a Llorca, Fariñas-Âlvarez y Delgado-Rodríguez $^{26}$, este contexto concuerda con el escenario de alta prevalencia de exposición y bajos riesgos relativos (RR), por lo que las medidas a implementar incluyen la instauración de intervenciones generales que beneficien a toda la población y la identificación de grupos de riesgo.

En este contexto resulta interesante analizar cuáles son los posibles efectos futuros de la actual ley sobre composición de los alimentos y su publicidad, en especial en el caso del consumo de sal, dado la dificultad de mantener un consumo reducido de sal con intervenciones de tipo individual ${ }^{5,7-9}$. Se recomienda la generación de proyectos con metodología robusta para el estudio de este tema puntual, la profundización de la respuesta a sal en la población y la identificación de sujetos de riesgo. 
Agradecimientos: A la Dra. Marcia Erazo, por haber sido tutora de la tesis de magíster de la que se desprende esta publicación.

\section{Referencias}

1. OMS. Informe sobre la situación mundial de las enfermedades no transmisibles 2014 [Internet]. WHO [citado el 21 de noviembre de 2017]. Disponible en: http://www. who.int/nmh/publications/ncd-status-report-2014/es/

2. WHO. Raised blood pressure [Internet]. WHO [citado el 11 de abril de 2016]. Disponible en: http://www.who. $\mathrm{int} / \mathrm{gho} / \mathrm{ncd} /$ risk_factors/blood_pressure_prevalence_text/en/

3. OMS. Reducción del consumo de sal en la población. Informe de un foro y una reunión técnica de la OMS del 5 al 7 de Octubre del 2006 [Internet]. 2007 [citado el 11 de abril de 2016]. Disponible en: http://www.who. int/dietphysicalactivity/salt-report-SP.pdf

4. He FJ, Pombo-Rodrigues S, MacGregor GA. Salt reduction in England from 2003 to 2011: its relationship to blood pressure, stroke and ischaemic heart disease mortality. BMJ Open 2014; 4 (4): e004549.

5. He FJ, Li J, MacGregor GA. Effect of longer term modest salt reduction on blood pressure: Cochrane systematic review and meta-analysis of randomised trials. BMJ 2013; 346: f1325.

6. Joffres MR, Campbell NRC, Manns B, Tu K. Estimate of the benefits of a population-based reduction in dietary sodium additives on hypertension and its related health care costs in Canada. Can J Cardiol 2007; 23 (6): 437-43.

7. Aburto NJ, Ziolkovska A, Hooper L, Elliott P, Cappuccio FP, Meerpohl JJ. Effect of lower sodium intake on health: systematic review and meta-analyses. PubMed Health [Internet]. 2013 [citado el 4 de abril de 2016]; Disponible en: http://www.ncbi.nlm.nih.gov/books/ PMH0054761/

8. Mohan S, Campbell NRC. Salt and high blood pressure. Clin Sci 2009; 117 (1): 1-11.

9. He FJ, Campbell NRC, MacGregor GA. Reducing salt intake to prevent hypertension and cardiovascular disease. Rev Panam Salud Pública Pan Am J Public Health 2012; 32 (4): 293-300.

10. Kanbay M, Chen Y, Solak Y, Sanders PW. Mechanisms and consequences of salt sensitivity and dietary salt intake. Curr Opin Nephrol Hypertens 2011; 20 (1): 37-43.

11. O'Donnell MJ, Mente A, Smyth A, Yusuf S. Salt intake and cardiovascular disease: why are the data inconsistent? Eur Heart J 2013; 34 (14): 1034-40.

12. Tanaka T, Okamura T, Miura K, Kadowaki T, Ueshima $\mathrm{H}$, Nakagawa $\mathrm{H}$, et al. A simple method to estimate populational 24 -h urinary sodium and potassium ex- cretion using a casual urine specimen. J Hum Hypertens 2002; 16 (2): 97-103.

13. Pontificia Universidad Católica de Chile M. Encuesta Nacional de Salud (ENS) 2009-2010 [Internet]. 2009 [citado el 27 de abril de 2017]. Disponible en: http:// web.minsal.cl/portal/url/item/bcb03d7bc28b64dfe040010165012d23.pdf

14. Dal Grande E, Gill T, Wyatt L, Chittleborough CR, Phillips PJ, Taylor AW. Population attributable risk (PAR) of overweight and obesity on chronic diseases: South Australian representative, cross-sectional data, 2004-2006. Obes Res Clin Pract 2009; 3 (3): I-IV.

15. Fleiss JL. Inference about population attributable risk from cross-sectional studies. Am J Epidemiol 1979; 110 (2): 103-4.

16. du Cailar G, Mimran A. Non-pressure-related effects of dietary sodium. Curr Hypertens Rep 2009; 11 (1): 12-7.

17. Drenjančević-Perić I, Jelaković B, Lombard JH, Kunert MP, Kibel A, Gros M. High-salt diet and hypertension: focus on the renin-angiotensin system. Kidney Blood Press Res 2011; 34 (1): 1-11.

18. Majid DSA, Prieto MC, Navar LG. Salt-Sensitive Hypertension: Perspectives on Intrarenal Mechanisms. Curr Hypertens Rev 2015; 11 (1): 38-48.

19. Richardson SI, Freedman BI, Ellison DH, Rodriguez CJ. Salt sensitivity: a review with a focus on non-Hispanic blacks and Hispanics. J Am Soc Hypertens JASH 2013; 7 (2): 170-9.

20. Leeuw PW de, Kroon AA. Salt and Sensitivity. Hypertension 2013; 62 (3): 461-2.

21. Oberleithner H, Wilhelmi M. Vascular glycocalyx sodium store-determinant of salt sensitivity? Blood Purif. 2015; 39 (1-3): 7-10.

22. AlGhatrif M, Wang M, Fedorova OV, Bagrov AY, Lakatta EG. The Pressure of Aging. Med Clin North Am 2017; 101 (1): 81-101.

23. Bursztyn M, Ben-Dov IZ. Sex differences in salt-sensitivity risk approximated from ambulatory blood pressure monitoring and mortality. J Hypertens 2013; 31 (5): 900-5.

24. Kelly TN, Rebholz CM, Gu D, Hixson JE, Rice TK, Cao J, et al. Analysis of Sex Hormone Genes Reveals Gender Differences in the Genetic Etiology of Blood Pressure Salt Sensitivity: The GenSalt Study. Am J Hypertens 2013; 26 (2): 191-200.

25. Benichou J. A review of adjusted estimators of attributable risk. Stat Methods Med Res 2001; 10 (3): 195-216.

26. Fracción atribuible poblacional: cálculo e interpretación. Gaceta Sanitaria [Internet] [citado el 4 de abril de 2016]. Disponible en: http://www.gacetasanitaria.org/es/fraccion-atribuible-poblacional-calculo-e/articulo-resumen/ S0213911101715191/. 